\title{
Dynamical chiral-symmetry breaking and exotic quark representations
}

\author{
J. C. Montero \\ Instituto de Física, Universidade de São Paulo, Caixa Postal 20516, Codigo de Enderecamento Postal 01498 São Paulo, \\ São Paulo, Brazil \\ V. Pleitez \\ Instituto de Física Teórica-Universidade Estadual Paulista, Rua Pamplona, 145, 01405 São Paulo, São Paulo, Brazil
}

(Received 16 February 1988)

\begin{abstract}
Using the effective potential for composite operators to study the chiral-symmetry-breaking transition in QCD, we examine a conjecture made by Marciano concerning the mass scale at which such transition occurs for exotic quark representations.
\end{abstract}

Some years ago Marciano ${ }^{1}$ speculated about the existence of exotic new species of quarks belonging to a higher representation of $\mathrm{SU}(3)$ in addition to the usual 3plets that are needed to describe the hadronic sector. In this case no extrastrong interaction based on an unbroken gauge group $\mathrm{SU}(N)$ will be introduced as in the hypercolor scenario. ${ }^{2,3}$

The basis of Marciano's conjecture is that the quantity

$$
C_{2}(R) \alpha_{s}(\mu),
$$

with $C_{2}(R)$ being the quadratic $\mathrm{SU}(3)$ Casimir invariant, is a measure of the effective color flux linking a quarkantiquark pair at mass scale $\mu$. As the Casimir invariants of the $R$ representation are bigger for the exotic representation than for the fundamental representation, they interact more strongly than ordinary quarks.

If $\mu_{R}$ and $\mu_{3}$ are the mass scales of the $R(R \neq 3)$ sector and 3-plet sector, respectively, at which the chiralsymmetry breaking occurs the question is as follows: can $\alpha_{s}\left(\mu_{R}\right)$ differ from $\alpha_{s}\left(\mu_{3}\right)$ ? In Ref. 1, two extreme possibilities were treated: (i) chiral symmetry is sector independent and (ii) chiral symmetry is sector dependent but such that

$$
C_{2}(R) \alpha_{s}\left(\mu_{R}\right)=C_{2}(3) \alpha_{s}\left(\mu_{3}\right) .
$$

The first possibility is not an interesting one, because it does not explain flavor-symmetry breaking in the Weinberg-Salam model. The second one, on the other hand, should allow a mass-scale hierarchy.

In this work we shall check if condition (2) is realized calculating $\alpha_{s}\left(\mu_{R}\right)$ and $\alpha_{s}\left(\mu_{3}\right)$ independently. For this we describe the chiral phase transformation using the effective potential for composite operators proposed some years ago for the authors of Ref. 4 and that have been used recently to study chiral-symmetry breaking in vectorlike theories. $^{5-9}$ There are some difficulties with this formalism. ${ }^{6,10}$ However, we hope that the critical coupling constants are not affected by these problems.

The effective potential derived in Ref. 4 has the form

$$
V(G)=-i \int \frac{d^{4} p}{(2 \pi)^{4}} \operatorname{Tr}\left(\ln S^{-1} G-S^{-1} G+1\right)+V_{2}(G),
$$

where $G$ is the complete propagator of fermions and $S$ the respective bare propagator. $V_{2}(G)$ is the sum of all twoparticle-irreducible vacuum diagrams. We calculate $V(G)$ in the usual approximation: $:^{5-9}$

$$
i G^{-1}=\not p-\Sigma(p)
$$

with $^{5,9}$

$\Sigma(p)=\phi, \quad p \leq p_{c}$

$$
=\phi \frac{p_{c}^{2}}{p^{2}}\left(\ln \frac{p_{c}}{\Lambda}\right)^{1-A / 2}\left(\ln \frac{P}{\Lambda}\right)^{A / 2-1}, p \geq p_{c},
$$

where

$$
A=18 C_{2}(R) /\left[11 N-4 n_{f} T(R)\right],
$$

$p_{c}$ is a momentum defining the infrared region and $\Lambda \approx 200 \mathrm{MeV}$ is the QCD scale. In (5), $\phi$ will be considered a variational parameter. We use, for the running coupling constant, ${ }^{11}$

$$
\begin{aligned}
\bar{g}^{2}(p, k) & =\bar{g}^{2}(p), \quad p>k \\
& =\bar{g}^{2}(k), \quad k>p
\end{aligned}
$$

with

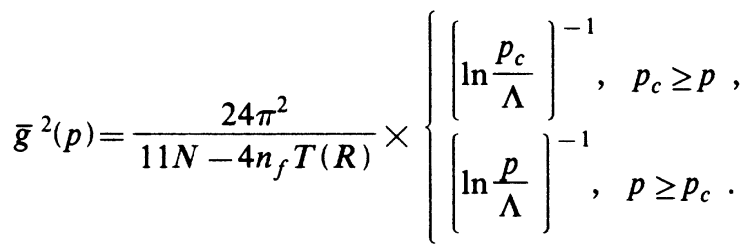

Introducing

$$
t=\frac{\Lambda^{2}}{p^{2}}, \quad z=\frac{p_{c}}{\Lambda}, \quad v=\frac{\phi}{\Lambda}
$$

and

$$
\widetilde{g}_{c}^{2}=(\ln z)^{-1}, \quad \widetilde{g}^{2}(t)=2\left[\ln \frac{1}{t}\right)^{-1}
$$

we can use (5)-(9) in (3) in order to obtain $V$ as a function of $v=\phi / \Lambda$. In this work we are only interested in deter- 
mining the critical value for $z\left(z_{c}\right)$. A necessary condition to have a transition is that there is a change of sign in the effective potential as we change the parameters of the theories. This fact can be seen already in the limit $v \ll<$. Making a tedious but straightforward algebra ${ }^{6-8}$ we obtain

$$
16 \pi^{2} \Lambda^{-4} V(v<<1)=v^{2} n_{f} d(R) F\left(z, n_{f}, C_{2}(R)\right)
$$

with

$$
\begin{aligned}
F\left(z, n_{f}, C_{2}(R)\right)= & 2 z^{2}+4 I_{1} \\
& -\frac{72 C_{2}(R)}{4\left(11 N-2 n_{f}\right)}\left[\left(\widetilde{g}_{c}^{2}+2 I_{2}\right) z^{2}+4 I_{3}\right]
\end{aligned}
$$

and with

$$
\begin{aligned}
& I_{1}=\frac{z^{4}}{2}(2 \ln z)^{2-A} \Gamma(A-1,2 \ln z), \\
& I_{2}=z^{2}(2 \ln z)^{1-A / 2} \Gamma\left(\frac{A}{2}-1,2 \ln z\right), \\
& I_{3}=\frac{-z^{4}}{A}(2 \ln z)^{2-A} \Gamma(A-1,2 \ln z)+\frac{2 z^{2} \ln z}{A} I_{2}
\end{aligned}
$$

with $\Gamma(\alpha, x)$ an incomplete gamma function. The critical value for $z\left(z_{c}\right)$ is such that $F\left(z_{c}, n_{f}, C_{2}(R)\right)=0$. The expression (11) can be calculated numerically. We have done it using the different representation-dependent parameters. For example, for $n_{f}=6$, QCD can accommodate just two 6-plets and still retain asymptotic freedom. ${ }^{1}$ For the last representation we have $C_{2}(6)=\frac{10}{3}, d(R)=6$, $T(R)=\frac{5}{2}$.

The critical value $z_{c}$ for the 3-plets representation is $z_{c}=1.88$ and for the 6-plets is 21.51. In Table I we show the values of $C_{2}(6) \alpha_{s}\left(\mu_{6}\right)$ for those representations as well as the relation between the two mass scales at which chiral-symmetry breaking occurs for the $R=3$ and 6 given by the respective values of $z_{c}$. From the values shown in Table I we see that relation (2) is satisfied within $17 \%$ of error but contrary to what is expected by Marci-
TABLE I. The values of $\alpha_{c} C_{2}$ for $R=3$, with $n_{f}(3)=6$, and $R=6$, with $n_{f}(6)=2$, and the corresponding ratio $\mu_{6} / \mu_{3}$.

\begin{tabular}{lrccc}
\hline \hline$R$ & $C_{2}(R)$ & $\alpha_{c}$ & $\alpha_{c} C_{2}(R)$ & $\mu_{6} / \mu_{3}$ \\
\hline 3 & $4 / 3$ & 1.42 & 1.89 & 11.44 \\
6 & $10 / 3$ & 0.47 & 1.58 & 11.44 \\
\hline \hline
\end{tabular}

ano the strong-coupling regime for the fundamental representation implies the weak-coupling regime for exotic quarks. Note also that $\mu_{6} / \mu_{3} \approx 10$; that means that the chiral-symmetry-breaking scale for the 6-plets is of the order of $1 \mathrm{GeV}$ and that there is no hierarchy between these mass scales. We recall that the analysis made in Ref. 1 was based on the hypothesis that $\alpha_{c}\left(\mu_{3}\right)$ is small enough to start trusting perturbation theory. The effective potential for composite operators we have used with the ansatz (5) may be sensitive, in a nontrivial way, to nonperturbative effects.

The values shown in Table I have only a qualitative status with respect to what is really realized. For example, with only a 6-plet we obtain $z_{c} \simeq 4.89$ [or $\left.\alpha_{c}(6) \simeq 0.52\right]$ and $C_{2}(6) \alpha_{s}(6) \simeq 1.72$; that is, relation (2) is satisfied within $9 \%$ of error.

We have also tried higher representations and found their critical couplings. Obviously to take them seriously we must look for a larger group in which to embed a QCD method that can accommodate these representations. For example, with one 8-plet we obtain $z_{c}=4.7434$ $\left(\alpha_{c}=0.58\right)$ and relation (2) is satisfied within $7 \%$ of error.

Only for the case of three 6-plets or one 10-plet does a large mass hierarchy $\mu_{6,10} / \mu_{3} \gg 1$ arise.

In this case we must also look for a group larger than $\mathrm{SU}(3)$. This could be the case in grand unified theories as was also suggested by Marciano. ${ }^{1}$

This work was partially supported by the Financiadora de Estudos e Projetos (Rio de Janeiro). J.C.M. thanks the Conselho Nacional de Desenvolvimento Científico e Technológico (Brasília) for financial support and the Instituto de Física Teórica-Universidade Estadual Paulista for their kind hospitality.
${ }^{1}$ W. J. Marciano, Phys. Rev. D 21, 2425 (1980).

${ }^{2}$ S. Weinberg, Phys. Rev. D 13, 974 (1976); 19, 1277 (1979).

${ }^{3}$ L. Susskind, Phys. Rev. D 20, 2619 (1979).

${ }^{4}$ J. M. Cornwall, R. Jackiw, and E. Tomboulis, Phys. Rev. D 10, 2428 (1974).

${ }^{5}$ P. Castorina and S-Y. Pi, Phys. Rev. D 31, 411 (1985).

${ }^{6}$ V. P. Gusynin and Yu. A. Sitenko, Z. Phys. C 29, 547 (1985).

${ }^{7}$ J. C. Montero and V. Pleitez, Phys. Rev. D 35, 2579 (1987).
${ }^{8}$ J. C. Montero and V. Pleitez, Report No. IFT/P-25/87 (unpublished).

${ }^{9}$ R. Casalbuoni et al., Phys. Lett. 150B, 295 (1985).

${ }^{10}$ R. W. Haymaker and T. Matsuki, Phys. Rev. D 33, 1137 (1986).

${ }^{11}$ K. Higashijima, Phys. Lett. 124B, 257 (1983); Phys. Rev. D 29, 1928 (1984). 